\title{
Association Between Dementia and Clinical Outcome After COVID-19: A Nationwide Cohort Study with Propensity Score Matched Control in South Korea
}

\section{Sheng-Min Wang}

Department of Psychiatry, Yeouido St. Mary's Hospital, College of Medicine, The Catholic University of Korea, Seoul

\section{Si-Hyun Park}

Department of Pharmacology, College of Medicine, The Catholic University of Korea, Seoul Nak-Young Kim

Department of Psychiatry, Yeouido St. Mary's Hospital, College of Medicine, The Catholic University of Korea, Seoul

\section{Dong Woo Kang}

Department of Psychiatry, Seoul St. Mary's Hospital, College of Medicine, The Catholic University of Korea, Seoul

\section{Hae-Ran $\mathrm{Na}$}

Department of Psychiatry, Yeouido St. Mary's Hospital, College of Medicine, The Catholic University of Korea, Seoul

\section{Yoo Hyun Um}

Department of Psychiatry, St. Vincent Hospital, Suwon, Korea, College of Medicine, The Catholic University of Korea, Seoul

\section{Seunghoon Han}

Department of Pharmacology, College of Medicine, The Catholic University of Korea, Seoul

\section{Sung-Soo Park ( $\square$ sspark@catholic.ac.kr)}

Department of Hematology, Seoul St. Mary's Hospital, College of Medicine, The Catholic University of Korea, Seoul

\section{Hyun Kook Lim}

Department of Psychiatry, Yeouido St. Mary's Hospital, College of Medicine, The Catholic University of Korea, Seoul

\section{Research Article}

Keywords: SARS-CoV-2, COVID-19, Dementia, Mortality 
Posted Date: December 15th, 2020

DOI: https://doi.org/10.21203/rs.3.rs-121469/v1

License: (c) (i) This work is licensed under a Creative Commons Attribution 4.0 International License. Read Full License 


\section{Abstract}

Despite a high prevalence of dementia in older adults hospitalized with severe acute respiratory syndrome coronavirus 2 infection (COVID-19), research investigating association between preexisting diagnoses of dementia and prognosis of COVID-19 is scarce. We explored a nationwide cohort with a total of 2,800 subjects older than 50 years who were diagnosed with COVID-19 between January and April 2020. Among them, 223 patients had underlying dementia (dementia group). We matched 1:1 for each dementia-non-dementia group pair yielding 223 patients without dementia (no dementia group) using propensity score matching. The primary outcome measure was group difference in mortality after COVID-19. Mortality rate after COVID-19 were significantly higher in dementia group than in no dementia group (33.6\% vs. $20.2 \%$, $\mathrm{p}=0.002$ ). In addition, dementia group had higher proportion of patients requiring invasive ventilatory support than no dementia group ( $34.1 \%$ vs. $22.0 \%, p=0.006)$. Multivariable analysis showed that dementia group had a higher risk of mortality than no dementia group (odds ratio $=3.05$, $p<0.001$ ). We also found that patients in dementia group had a higher risk of needing invasive ventilatory support than those in no dementia group. Our results suggest that system including strengthen quarantines are required for patients with dementia during the COVID-19 pandemic.

\section{Introduction}

An outbreak caused by severe acute respiratory syndrome coronavirus 2 (SARS-CoV-2), or so called COVID-19, first occurred in Wuhan, Hubei Province, China at the end of 2019 ${ }^{1}$. Thereafter, COVID-19 has spread worldwide and become global pandemic. Numerous studies identified diverse risk factors associated with severity and mortality after SARS-CoV-2 infections ${ }^{2,3}$. Risk factors varied depending on the studies, but it is generally acknowledged that history of chronic medical diseases and conditions reducing general immunity are associated worse treatment outcome in patients with COVID-19. Studies showed that older age (>65), cigarette smoking, use of anti-inflammatory biological agents, and having pre-existing disease including pulmonary disease, allergic rhinitis, asthma, hypertension, cardiovascular disease, chronic kidney disease, diabetes, obesity, malignancy, chronic HIV infection, and transplantation could be possible risk factors for severe COVID-193-5.

Patients with dementia are known to have a higher risk of developing respiratory infection. Pneumonia is one of the leading causes of acute care hospitalization in patients with dementia ${ }^{6}$. Moreover, pneumonia is the most common cause of death in patients with dementia ${ }^{7}$. More than 50 million people worldwide are suffering from dementia, and one new case occurs every 3 seconds ${ }^{8}$. Thus, the "double hit" of dementia and COVID-19 pandemic could result in a more detrimental effect in people living with dementia $^{9}$. In line with this, Alzheimer's Disease International (ADI) recently reported that 85 percent of all COVID-19 deaths in Canada occurred in long term care facilities, where two thirds of people had dementia ${ }^{10}$. 
There have been limited researches investigating association between preexisting diagnoses of dementia and prognosis of COVID-19. A recent study based on UK Biobank Community Cohort showed that dementia was associated with the largest risk increase (Odds ratio $(O R)=7.30)$ for COVID-19 hospitalization and death ${ }^{11}$. However, among 507 patients included in the COVID-19 group, the number of patients diagnosed with dementia were very small $(n=14)$. Another study identified that dementia was associated with increased hospital mortality ${ }^{12}$, but the main objective of the research was to characterize the clinical features of patients admitted to hospital with COVID-19 rather than focusing on the impact of dementia in the treatment outcome of COVID-19.

We aimed to investigate treatment outcome of patients with dementia after COVID-19. Using a nationwide cohort in South Korea (hereafter Korea), we compared treatment outcome after COVID-19 infection between patients having underlying dementia with those not having dementia. We hypothesized that underlying dementia might increase the risk of patients having more severe outcomes after COVID19 infection.

\section{Results}

\section{Baseline characteristics}

A total of 5,628 subjects were diagnosed with SARS-CoV-2 between January and April 2020. After excluding seven patients who were diagnosed with SARS-CoV-2 after death, 27 patients who did not have disease course after SARS-CoV-2, and 2,794 patients who were younger than age of 50 years, a total of 2,800 patients were initially available for analysis. Among them, we identified 223 patients (dementia group) with dementia and 2,577 patients without dementia. Subsequently, we matched 1:1 for each dementia-non-dementia group pair yielding 223 patients in non-dementia group (Fig. 1). After matching propensity score of the dementia group with that of the no dementia group, the two groups did not differ in their baseline patient characteristics (Table 1). 
Table 1

Baseline demographic and clinical characteristics

\begin{tabular}{|c|c|c|c|}
\hline \multirow[t]{2}{*}{ Variables } & \multicolumn{2}{|l|}{ Groups } & \multirow{2}{*}{$\begin{array}{l}p- \\
\text { value }\end{array}$} \\
\hline & $\begin{array}{l}\text { Dementia }(n= \\
223)\end{array}$ & $\begin{array}{l}\text { No Dementia }(n= \\
223)\end{array}$ & \\
\hline Age, no (\%) & & & 0.949 \\
\hline $50-59$ years & $9(4.0 \%)$ & $7(3.1 \%)$ & \\
\hline $60-69$ years & $22(9.9 \%)$ & $21(9.4 \%)$ & \\
\hline $70-79$ years & $52(23.3 \%)$ & $55(24.7 \%)$ & \\
\hline$\geq 80$ years & $140(62.8 \%)$ & $140(62.8 \%)$ & \\
\hline Gender, no (\%) & & & 0.272 \\
\hline Female & $153(68.6 \%)$ & $141(63.2 \%)$ & \\
\hline Male & $70(31.4 \%)$ & $82(36.8 \%)$ & \\
\hline Systolic blood pressure, no (\%) & & & 0.686 \\
\hline$<120 \mathrm{mmHg}$ & $54(24.2 \%)$ & $51(22.9 \%)$ & \\
\hline $120-129 \mathrm{mmHg}$ & $36(16.1 \%)$ & $26(11.7 \%)$ & \\
\hline $130-139 \mathrm{mmHg}$ & $35(15.7 \%)$ & $32(14.3 \%)$ & \\
\hline $140-159 \mathrm{mmHg}$ & $65(29.1 \%)$ & $75(33.6 \%)$ & \\
\hline$\geq 160 \mathrm{mmHg}$ & $30(13.5 \%)$ & $36(16.1 \%)$ & \\
\hline Diastolic blood pressure, no (\%) & & & 0.829 \\
\hline$<80 \mathrm{mmHg}$ & $105(47.1 \%)$ & $110(49.3 \%)$ & \\
\hline $80-89 \mathrm{mmHg}$ & $67(30.0 \%)$ & $59(26.5 \%)$ & \\
\hline $90-99 \mathrm{mmHg}$ & $35(15.7 \%)$ & $33(14.8 \%)$ & \\
\hline$\geq 100 \mathrm{mmHg}$ & $13(5.8 \%)$ & $18(8.1 \%)$ & \\
\hline Heart rate, (bests/min, mean \pm SD) & $80.7 \pm 17.5$ & $82.3 \pm 15.3$ & 0.326 \\
\hline Body temperature $\left({ }^{\circ} \mathrm{C}\right.$, mean $\left.\pm \mathrm{SD}\right)$ & $36.8 \pm 0.6$ & $36.8 \pm 0.5$ & 0.765 \\
\hline \multicolumn{4}{|l|}{ Baseline presentation, no (\%), } \\
\hline Sputum (+) & $27(12.1 \%)$ & $33(14.8 \%)$ & 0.422 \\
\hline Fatigue (+) & $11(4.9 \%)$ & $10(4.5 \%)$ & 0.592 \\
\hline Dyspnea (+) & 37 (16.6\%) & 43 (19.3\%) & 0.453 \\
\hline
\end{tabular}




\begin{tabular}{|c|c|c|c|}
\hline \multirow[t]{2}{*}{ Variables } & \multicolumn{2}{|l|}{ Groups } & \multirow{2}{*}{$\begin{array}{l}p- \\
\text { value }\end{array}$} \\
\hline & $\begin{array}{l}\text { Dementia }(n= \\
\text { 223) }\end{array}$ & $\begin{array}{l}\text { No Dementia }(n= \\
\text { 223) }\end{array}$ & \\
\hline Altered mental status (+) & $8(3.6 \%)$ & $4(1.8 \%)$ & 0.308 \\
\hline Nausea/vomiting $(+)$ & $7(3.1 \%)$ & $7(3.1 \%)$ & 0.606 \\
\hline Diarrhea $(+)$ & $13(5.8 \%)$ & $21(9.4 \%)$ & 0.214 \\
\hline \multicolumn{4}{|l|}{ Comorbidity, no (\%) } \\
\hline Diabetes $(+)$ & $63(28.3 \%)$ & $69(30.9 \%)$ & 0.604 \\
\hline Hypertension (+) & $126(56.5 \%)$ & $124(55.6 \%)$ & 0.924 \\
\hline Heart failure $(+)$ & $12(5.4 \%)$ & $14(6.3 \%)$ & 0.840 \\
\hline Cardiac disease $(+)$ & $15(6.7 \%)$ & $20(9.0 \%)$ & 0.416 \\
\hline Asthma (+) & $8(3.6 \%)$ & $10(4.5 \%)$ & 0.810 \\
\hline Chronic obstructive lung disease $(+)$ & $8(3.6 \%)$ & $6(2.7 \%)$ & 0.786 \\
\hline Chronic renal disease $(+)$ & $10(4.5 \%)$ & $13(5.8 \%)$ & 0.668 \\
\hline Previous malignant disease ${ }^{\mathrm{a}}(+)$ & $5(2.2 \%)$ & $6(2.7 \%)$ & 0.999 \\
\hline Chronic hepatic disease $(+)$ & $6(2.7 \%)$ & $5(2.7 \%)$ & 0.999 \\
\hline Autoimmune disease (+) & $0(0.0 \%)$ & $0(0.0 \%)$ & \\
\hline \multicolumn{4}{|l|}{ Baseline hemogram } \\
\hline Hemoglobin, g/dL \pm SD & $12.1 \pm 1.9$ & $12.1 \pm 1.8$ & 0.855 \\
\hline Whole blood cell counts, $/ \mathrm{mm}^{3}$, mean $\pm \mathrm{SD}$ & $6,130 \pm 2,907$ & $6,487 \pm 3,169$ & 0.216 \\
\hline $\begin{array}{l}\text { Absolute lymphocyte counts, } / \mathrm{mm}^{3} \text {, mean } \pm \\
\text { SD }\end{array}$ & $1,322 \pm 691$ & $1,336 \pm 627$ & 0.827 \\
\hline Platelet, $/ \mathrm{mm}^{3}$, mean $\pm \mathrm{SD}$ & $207,468 \pm 80,951$ & $215,027 \pm 79,296$ & 0.320 \\
\hline
\end{tabular}

\section{Primary and secondary outcomes}

Mortality rate after SARS-CoV-2 infection were significantly higher in dementia group than in no dementia group (33.6\% vs $20.2 \%, p=0.002$ ). In addition, dementia group had higher proportion of patients requiring invasive ventilatory support than no dementia group (34.1\% vs $22.0 \%, p=0.006)$. However, time from to 
admission to death, length of hospitalization, and rate of patients needing oxygen supply did not significantly differ between the two groups (Table 2).

Table 2

Treatment outcomes of SARS-CoV-2

\begin{tabular}{|c|c|c|c|}
\hline \multirow[t]{2}{*}{ Outcomes } & \multicolumn{2}{|l|}{ Groups } & \multirow{2}{*}{$\begin{array}{l}p- \\
\text { value }\end{array}$} \\
\hline & $\begin{array}{l}\text { Dementia } \\
(n=223)\end{array}$ & $\begin{array}{l}\text { No Dementia, }(n= \\
\text { 223) }\end{array}$ & \\
\hline Death, no (\%) & $\begin{array}{l}75 \\
(33.6 \%)\end{array}$ & $45(20.2 \%)$ & $0.002 *$ \\
\hline $\begin{array}{l}\text { Days alive from admission to death in those who died, } \\
\text { mean } \pm S D\end{array}$ & $\begin{array}{l}14.0 \pm \\
11.5\end{array}$ & $15.5 \pm 11.9$ & 0.483 \\
\hline $\begin{array}{l}\text { Days needing hospitalization in those who recovered, } \\
\text { mean } \pm S D\end{array}$ & $\begin{array}{l}29.6 \pm \\
10.8\end{array}$ & $29.1 \pm 120$ & 0.714 \\
\hline Requiring oxygen supply, no (\%) & $\begin{array}{l}117 \\
(52.5 \%)\end{array}$ & $102(45.7 \%)$ & 0.185 \\
\hline Requiring invasive ventilative support, no (\%) & $\begin{array}{l}76 \\
(34.1 \%)\end{array}$ & $49(22.0 \%)$ & $0.006 *$ \\
\hline
\end{tabular}

Note. ${ }^{a}$ Any oxygen support more intensive than nasal cannula; SD, standard deviation; ${ }^{\star} p<.05$

\section{Prognostic parameters for mortality of COVID-19}

Univariable analysis showed that age, baseline heart rate, presentations including dyspnea and altered mental status, and baseline blood cell examination including hemoglobin and absolute lymphocyte counts were potential factors associated with mortality. In addition, comorbidities including dementia, diabetes, hypertension, and chronic renal disease were also identified as potential factors (supplementary table). In multivariable analysis using potential variables derived from the univariate analysis, we observed that dementia had significant impacts on survival ( $\mathrm{OR}=3.05,95 \% \mathrm{Cl}: 1.80-5.4, p<0.001)$ (Fig. 2). Other factors including age, heart rate, dyspnea, altered mental status, diabetes, chronic renal disease, and absolute lymphocyte counts were also significant factors associated with higher mortality after COVID-19 (Table 3). 
Table 3

Multivariable analysis of factors associated with event of death after SARS-CoV2 infection

\begin{tabular}{|lll|}
\hline Variable & Odd ratio $(95 \%$ Cl) & p-value \\
\hline Age & & \\
\hline$<80$ years & 1 & \\
\hline$\geq 80$ years & $3.40(1.91-6.28)$ & $<0.001^{\star}$ \\
\hline Heart rate, baseline & & \\
\hline$<110 /$ min & 1 & \\
\hline$\geq 110 /$ min & $4.22(1.49-12.32)$ & $0.007^{*}$ \\
\hline Dyspnea at presentation & & \\
\hline Not present & 1 & \\
\hline Present & $2.49(1.35-4.59)$ & $0.004^{\star}$ \\
\hline Altered mental status at presentation & & \\
\hline Not present & 1 & \\
\hline Present & $7.03(1.45-52.48)$ & $0.026^{*}$ \\
\hline Comorbidity & & \\
\hline Dementia, no & $1.63(0.96-2.81)$ & 0.073 \\
\hline Dementia, yes & 1 & \\
\hline Diabetes, no & 1 & \\
\hline Diabetes, yes & $3.05(1.80-5.3)$ & $<0.001^{\star}$ \\
\hline Hypertension, no & 1 & \\
\hline Hypertension, yes & $2.32(1.32-4.1)$ & 0.004 \\
\hline Chronic renal disease, no & 1 & \\
\hline Chronic renal disease, yes & & \\
\hline Hemoglobin at presentation & & \\
\hline$\geq 12.5 \mathrm{~g} / \mathrm{dL}$ & & \\
\hline$<12.5 \mathrm{~g} / \mathrm{dL}$ & & \\
\hline Absolute lymphocyte counts at presentation & & \\
\hline
\end{tabular}

Note. ${ }^{*} p<.05$ 


\begin{tabular}{|lll|}
\hline Variable & Odd ratio $(95 \% \mathrm{Cl})$ & $p$-value \\
\hline$\geq 1,000 / \mathrm{mm}^{3}$ & 1 & \\
\hline$<1,000 / \mathrm{mm}^{3}$ & $4.27(2.53-7.34)$ & $<0.001 *$ \\
\hline Note. ${ }^{*} p<.05$ & & \\
\hline
\end{tabular}

\section{Discussion}

We compared patients who had underlying dementia with those propensity score-matched patients not having dementia and analyzed their disease severity and survival outcome after SARS-CoV-2 infection. Our study showed that patients diagnosed with dementia have three times higher risk of mortality than patients without dementia in a nationwide cohort from Korea. In addition, higher proportion of dementia group required invasive ventilatory support than no dementia group.

In line with our findings, a previous study showed that dementia has the largest effect risk of mortality in adults aged older than 65 who were diagnosed with COVID-19 ${ }^{11}$. However, it is still unclear how underlying dementia could affect mortality and severity after COVID-19. Patients with dementia have a more difficulty expressing his or her somatic symptoms, and clinicians could easily mis-interpret them as cognitive or behavior symptoms of dementia ${ }^{13}$. Thus, important signs and symptoms associated with aggravation of COVID-19 could be unnoticed which could have delayed them from receiving timely treatment. In line with this hypothesis, in the elderly with dementia, delirium was one of the most frequent early presentation of COVID-19 $9^{14}$. In addition, dementia is the most important and leading risk factor for delirium $^{15}$, and delirium is known to increase the risk of mortality during an acute hospital admission ${ }^{16}$. Likewise, delirium was found to be independently associated with increased mortality in elderly adults diagnosed with COVID-1914.

Although the pathogenic mechanism of dementia on severe course of COVID-19 is still unexplainable, immune dysregulation might be an one of important pathophysiologies ${ }^{17}$. Evidences indicated that an uncontrolled over-production of soluble markers of inflammation, following immunologic alteration, is a major cause of acute respiratory distress syndrome in patients with COVID-19 ${ }^{18}$. Recent researches highlighted that aberrant activities of astrocytes and microglia could be important mediators of SARSCoV-2-induced neurological damage and cytokine storm ${ }^{19}$. Microglia and astrocyte activation are important hall marks in the pathophysiology Alzheimer's disease (the most common cause of dementia) $)^{20,21}$. Thus, patients with dementia might be at increased risk of excessive production of cytokines than those without dementia. However, further work is needed to elucidate exact pathophysiological association between dementia and clinical outcome after COVID-19.

Days alive from admission to death, days of hospitalization before discharge, and rate of patients needing oxygen supply did not show significant differences between dementia and no dementia groups. 
A study comprising COVID-19 patients over 60 years of age showed that rapid disease progress was noted in the dead regardless of underlying conditions ${ }^{22}$. Moreover, almost all of the dead patients were initially critically ill (92.3\%). Thus, patients who died in our study might have also shown similarly rapid progress regardless of dementia presence. In line with this hypothesis, in both dementia and no dementia group, almost all of the dead patients were severely ill requiring invasive ventilatory support (>92\% for both groups).

In the other perspective, SARS-CoV-2 infection may accentuate pre-existing dementia ${ }^{23}$. SARS-CoV-2 may have both direct effects on the brain via viral infection and vascular cascade within in the brain indirect effects on the brain via immunological response of the host ${ }^{24}$. Recent findings showed that up to one third of COVID-19 patients have demonstrated neurological sequelae ${ }^{25}$. Thus, our results might justify that additional attention is needed when patients with COVID-19 have underlying dementia.

Our study has several strengths. Despite observational data confirming that there is a high prevalence of dementia in older adults hospitalized with COVID- $19^{26}$, no controlled study so far has specifically investigated effect of dementia in the clinical outcome of COVID-19. Dementia is a disease of old age and is also associated with a variety of comorbidities, so creating a study cohort and finding appropriate control could have been more difficult than other medical conditions ${ }^{11}$. We conducted a nationwide cohort study and first included all patients who were diagnosed with COVID-19 and received admission care at a designated hospital. Thereafter, patients who were diagnosed with dementia before COVID-19 were selected among the mother cohort. By doing so, we were also able to include 223 patients (all aged 50 years or above) having both dementia and COVID-19 and minimize selection bias and treatment setting effect. In addition, by using propensity score matching via multivariable methods, we were also able to find equal number of patients having similar baseline demographic and clinical characteristics to no dementia group.

This study contains several limitations. First, presence of underlying dementia was based on reports of the patients and/or the guardians (family members), so it is subject to recall bias. Likewise, patients with dementia group might have under-reported their other comorbidities which could have confounded our results by influencing propensity score. Second, we were unable to undertake cerebral imaging studies and objective cognitive testing. Therefore, it was not possible to investigate whether dementia subtype or severity of cognitive impairment had an effect in mortality or clinical outcome after COVID-19. Lastly, other important risk factors such as obesity, cigarette smoking, and chronic neurological disorders other than dementia were not included as parts of covariates.

In conclusion, using a large nationwide, propensity score matched cohort, we found that patients diagnosed with dementia have a higher risk of needing invasive ventilatory support and mortality than patients without dementia after COVID-19. Additional researches are needed to confirm our findings and investigate pathophysiological association between dementia and COVID-19. Lastly, our results suggest additional attentions are required for patients with dementia during the course of COVID-19 treatment. 


\section{Methods}

\section{Data Source}

Korea Disease Control \& Prevention Agency (KDCA), a public organization under the South Korean Ministry of Welfare and Health, is responsible for performing defense and monitoring activities to contain and prevent COVID-1927. All patients who were diagnosed with COVID-19 received mandatory and complimentary care either at a hospital or a medical facility ${ }^{28}$. While patients received medical care, the KDCA was able to collect all medical data related to diagnosis and treatment of COVID-19. In addition, all physicians in charge of patient care were required to complete a case report form developed by the KDCA which contained patients' demographic and epidemiological characteristics and their detailed medical, surgical, and psychiatric histories (http://icreat.nih.go.kr/). The final consolidated COVID-19 data, which were provided by the KDCA, consisted of 42 clinical variables and 5,628 observations.

\section{Study population}

The study population was defined as all individuals aged older than 50 years who were confirmed to have COVID-19 infection between January and April 2020. COVID-19 infection was defined as laboratory confirmation of a positive result using real-time reverse transcriptase polymerase chain reaction assay using the Korean Ministry of Food and Drug Safety approved kit ${ }^{29,30}$. Nasal and pharyngeal swabs were assessed for diagnosis of COVID-19, which was in accordance with World Health Organization guidelines ${ }^{31}$. Presence of dementia was defined based on the information collected from the case report form, which was completed by the physicians who treated patients. To ensure confidentiality, all patientrelated data were anonymized. The data was provided by KDCA, so the consent was waived by the KDCA. In addition, the institutional review board of Seoul St. Mary's Hospital, Seoul, Korea, also approved consent waiving and approved the current study (KC20ZADI0654)

\section{Outcomes measurements}

The primary outcome measure of this study was mortality difference after COVID-19 between patients with underlying dementia (dementia group) and patients without dementia (no dementia group). As secondary outcome measures, we also compared days from admission to death, length of hospital stay before discharge, rate of patients requiring any oxygen supply, and rate of patients requiring invasive ventilatory support including mechanical ventilatory care and tracheostomy.

\section{Propensity matching score}

To account for the non-randomized treatment allocation, the propensity score was used to select a matching patient among patients not diagnosed with dementia. The missing values of all continuous dependent variables need to be calculated prior to applying the propensity score matching. Data was imputed by regressing each variable in turn against all other variables and then predicting missing data for the dependent variable using the fitted forest. Nearest neighbor matching was used to match patients 
in a non-dementia cohort with the closest propensity score for each patient in the dementia group. Age, gender, systolic blood pressure, diastolic blood pressure, comorbidity, and baseline hematological variables were used as covariates for matching to generate a mother cohort with a 1:1 ratio for the dementia and non-dementia groups.

\section{Statistical analysis}

Continuous and categorical variables were denoted by mean \pm standard deviation and $n(\%)$ in each dementia and non-dementia group, respectively. Difference between the dementia and non-depression group in baseline demographic and clinical characteristics were compared using Student's-T test for continuous and $\chi 2$ test for categorical variables. Multivariable logistics regression model was used to explore the risk factor for all variables that has $p$-value $<0.05$ in univariate analysis. A two tailed test with alpha $<0.05$ was considered to be statistically significant for overall analysis. For all statistical analysis, we used R statistical software (ver. 3.6.1, R Foundation for Statistical Computing, Vienna, Austria, 201907-05).

\section{Declarations}

\section{Data availability statement}

The data that support the findings of this study are available from ["is.cdc.go.kr"] but restrictions apply to the availability of these data, which were used under license for the current study, and so are not publicly available. Data are however available from the authors upon reasonable request and with permission of [Korea Disease Control \& Prevention Agency].

\section{Acknowledgments}

We acknowledge all the health-care workers involved in the diagnosis and treatment of COVID-19 patients in South Korea. We thank Korea Disease Control \& Prevention Agency, National Medical Center and the Health Information Manager in hospitals for their effort in collecting the medical records.

\section{Author contributions}

Sheng-Min Wang, Si-Hyun Park, Sung-Soo Park, and Hyun Kook Lim designed the study, analyzed and interpreted data, and draft manuscript. Seunghoon Han, contributed to project design, data collection, statistical analysis, and management; Nak-Young Kim, Dong Woo Kang, Hae-Ran Na, and Yoo Hyun Um were involved in manuscript revision. All authors have read and approved the revised manuscript.

\section{Funding}

This work was supported by the National Research Foundation of Korea (NRF) grant funded by the Korea government (MSIT) (NRF-2019R1C1C1011664).

\section{Competing interests}


The author(s) declare no competing interests.

\section{References}

1. Wiersinga, W. J., Rhodes, A., Cheng, A. C., Peacock, S. J. \& Prescott, H. C. Pathophysiology, Transmission, Diagnosis, and Treatment of Coronavirus Disease 2019 (COVID-19): A Review. JAMA 324, 782-793, doi:10.1001/jama.2020.12839 (2020).

2. Tian, W. et al. Predictors of mortality in hospitalized COVID-19 patients: A systematic review and meta-analysis. J Med Virol, doi:10.1002/jmv.26050 (2020).

3. Li, X. et al. Risk factors for severity and mortality in adult COVID-19 inpatients in Wuhan. J Allergy Clin Immunol 146, 110-118, doi:10.1016/j.jaci.2020.04.006 (2020).

4. Yang, J. M. et al. Allergic disorders and susceptibility to and severity of COVID-19: A nationwide cohort study. J Allergy Clin Immunol, doi:10.1016/j.jaci.2020.08.008 (2020).

5. Zhou, F. et al. Clinical course and risk factors for mortality of adult inpatients with COVID-19 in Wuhan, China: a retrospective cohort study. Lancet 395, 1054-1062, doi:10.1016/S01406736(20)30566-3 (2020).

6. Liao, K. M., Lin, T. C., Li, C. Y. \& Yang, Y. H. Dementia Increases Severe Sepsis and Mortality in Hospitalized Patients With Chronic Obstructive Pulmonary Disease. Medicine (Baltimore) 94, e967, doi:10.1097/MD.0000000000000967 (2015).

7. Brunnstrom, H. R. \& Englund, E. M. Cause of death in patients with dementia disorders. Eur J Neurol 16, 488-492, doi:10.1111/j.1468-1331.2008.02503.x (2009).

8. Alzheimer's Disease International. World Alzheimer's report 2019: attitudes to dementia. September, h. w. a. c. u. r. W. p. a. S., 2020).

9. Wang, H. et al. Dementia care during COVID-19. Lancet 395, 1190-1191, doi:10.1016/S01406736(20)30755-8 (2020).

10. Alzheimer's Disease International. News Release. September, C.-d. d. a. p. w. d., targeted response urgently needed. https://www.alz.co.uk/media/010920 (accessed September 30, 2020).

11. Atkins, J. L. et al. Preexisting Comorbidities Predicting COVID-19 and Mortality in the UK Biobank Community Cohort. J Gerontol A Biol Sci Med Sci, doi:10.1093/gerona/glaa183 (2020).

12. Docherty, A. B. et al. Features of 20133 UK patients in hospital with covid-19 using the ISARIC WHO Clinical Characterisation Protocol: prospective observational cohort study. BMJ 369, m1985, doi:10.1136/bmj.m1985 (2020).

13. Achterberg, W. P. et al. Pain management in patients with dementia. Clin Interv Aging 8, 1471-1482, doi:10.2147/CIA.S36739 (2013).

14. Poloni, T. E. et al. Prevalence and prognostic value of Delirium as the initial presentation of COVID-19 in the elderly with dementia: An Italian retrospective study. EClinicalMedicine 26, 100490, doi:10.1016/j.eclinm.2020.100490 (2020). 
15. Fong, T. G., Davis, D., Growdon, M. E., Albuquerque, A. \& Inouye, S. K. The interface between delirium and dementia in elderly adults. Lancet Neurol 14, 823-832, doi:10.1016/S1474-4422(15)00101-5 (2015).

16. Diwell, R. A., Davis, D. H., Vickerstaff, V. \& Sampson, E. L. Key components of the delirium syndrome and mortality: greater impact of acute change and disorganised thinking in a prospective cohort study. BMC Geriatr 18, 24, doi:10.1186/s12877-018-0719-1 (2018).

17. Fotuhi, M., Mian, A., Meysami, S. \& Raji, C. A. Neurobiology of COVID-19. J Alzheimers Dis 76, 3-19, doi:10.3233/JAD-200581 (2020).

18. Coperchini, F., Chiovato, L., Croce, L., Magri, F. \& Rotondi, M. The cytokine storm in COVID-19: An overview of the involvement of the chemokine/chemokine-receptor system. Cytokine Growth Factor Rev 53, 25-32, doi:10.1016/j.cytogfr.2020.05.003 (2020).

19. Vargas, G. et al. Severe acute respiratory syndrome coronavirus 2 (SARS-CoV-2) and glial cells: Insights and perspectives. Brain Behav Immun Health 7, 100127, doi:10.1016/j.bbih.2020.100127 (2020).

20. Heneka, M. T. et al. Neuroinflammation in Alzheimer's disease. Lancet Neurol 14, 388-405, doi:10.1016/S1474-4422(15)70016-5 (2015).

21. Perez-Nievas, B. G. \& Serrano-Pozo, A. Deciphering the Astrocyte Reaction in Alzheimer's Disease. Front Aging Neurosci 10, 114, doi:10.3389/fnagi.2018.00114 (2018).

22. Wang, L. et al. Coronavirus disease 2019 in elderly patients: Characteristics and prognostic factors based on 4-week follow-up. J Infect 80, 639-645, doi:10.1016/j.jinf.2020.03.019 (2020).

23. Killen, A. et al. The challenges of COVID-19 for people with dementia with Lewy bodies and family caregivers. Int J Geriatr Psychiatry, doi:10.1002/gps.5393 (2020).

24. Needham, E. J., Chou, S. H., Coles, A. J. \& Menon, D. K. Neurological Implications of COVID-19 Infections. Neurocrit Care 32, 667-671, doi:10.1007/s12028-020-00978-4 (2020).

25. Carod-Artal, F. J. Neurological complications of coronavirus and COVID-19. Rev Neurol 70, 311-322, doi:10.33588/rn.7009.2020179 (2020).

26. International Severe Acute Respiratory and Emerging Infections Consortium (ISARIC). COVID-19 Report 2020. https://isaric.tghn.org/covid-19-clinical-research-resources/. Accessed September 30.

27. Sung, H. et al. Preparedness and Rapid Implementation of External Quality Assessment Helped Quickly Increase COVID-19 Testing Capacity in the Republic of Korea. Clin Chem 66, 979-981, doi:10.1093/clinchem/hvaa097 (2020).

28. Kang, J. et al. South Korea's responses to stop the COVID-19 pandemic. Am J Infect Contro/ 48, 1080-1086, doi:10.1016/j.ajic.2020.06.003 (2020).

29. WHO COVID-19 Case definition. Updated in Public health surveillance for COVID-19, published 7 August 2020., <https://www.who.int/publications/i/item/WHO-2019-nCoVSurveillance_Case_Definition-2020.1>( 
30. Korea Disease Control and Prevention Agency. Case definition and people subject to testing (As of June 25, 2020). <http://ncov.mohw.go.kr/en/baroView.do?

brdld=11\&brdGubun=112\&dataGubun=\&ncvContSeq=\&contSeq=\&board_id=\&gubunn= > (

31. Koh, D. \& Cunningham, A. C. Counting Coronavirus Disease 2019 (COVID-19) Cases: Case Definitions, Screened Populations and Testing Techniques Matter. Ann Acad Med Singap 49, 161-165 (2020).

Figures

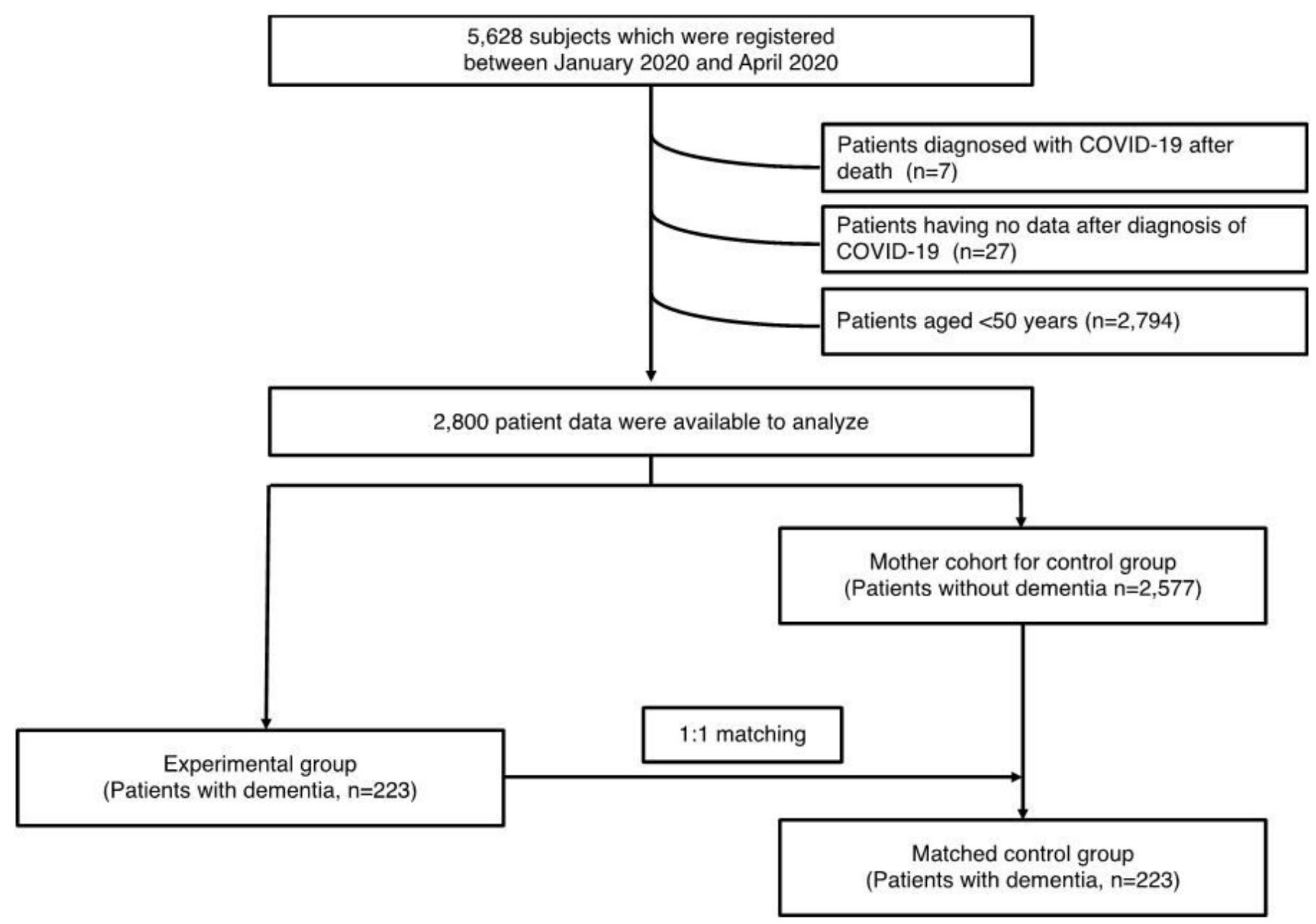

\section{Figure 1}

Flow chart depicting creation of study cohorts 


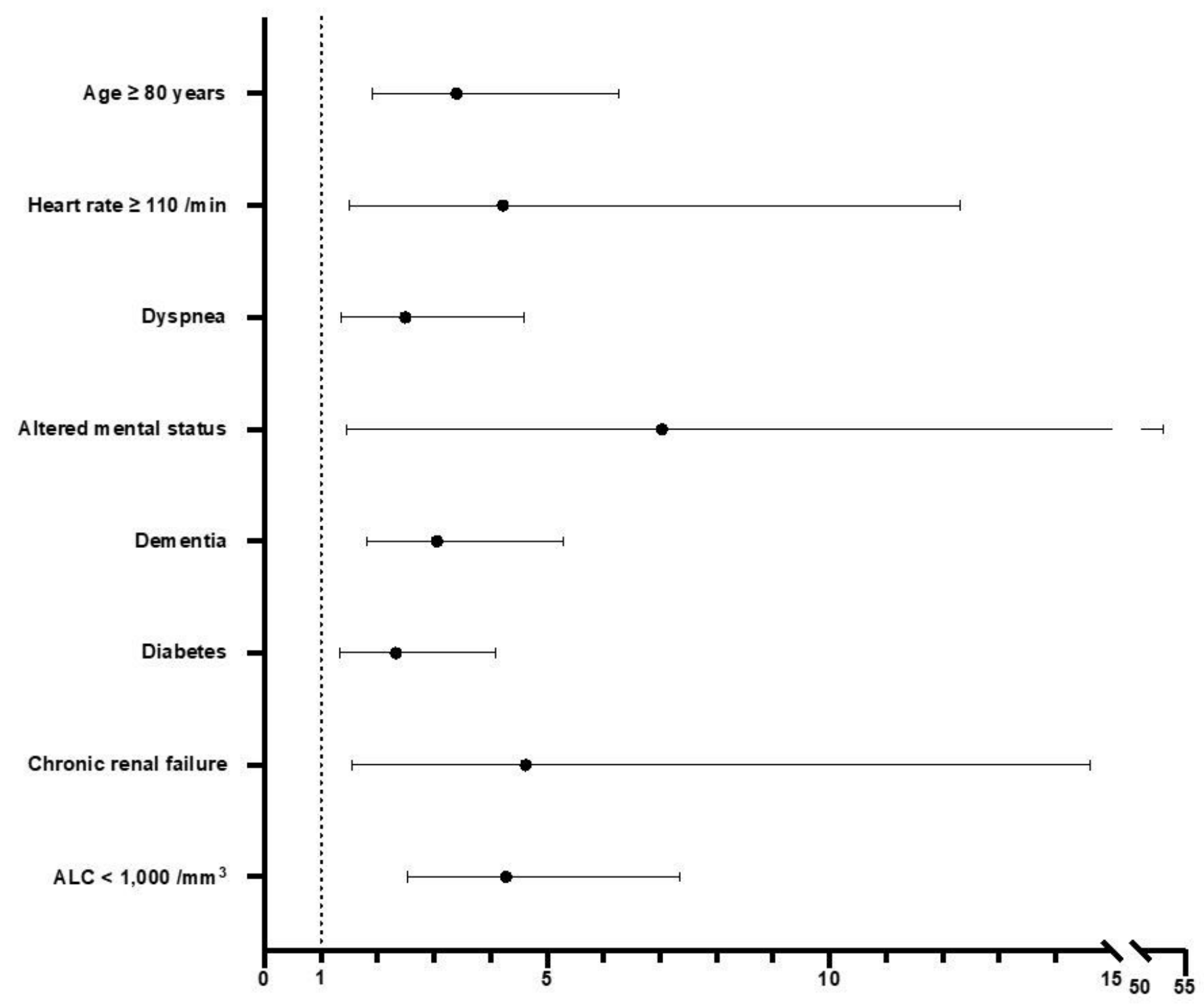

Odds ratio

Figure 2

Multivariable analysis of factors associated with event of death after SARS-CoV-2 infection

\section{Supplementary Files}

This is a list of supplementary files associated with this preprint. Click to download.

- Supplementaryinformationfile.pdf 